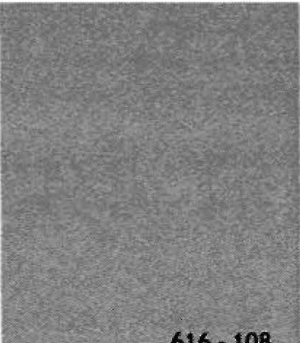

\title{
problemas de construcción y de explotación de los grandes hornos rotatorios por vía seca*
}

Se indican las consideraciones que han incitado a la industria del cemento a construir grandes unidades de hornos por vía seca. Se describen los métodos que hoy se encuentran en el mercado. Se compara la economía térmica de algunos hornos por vía seca, dedicando especial atención a la recuperación de calor de los gases y al funcionamiento del enfriador. Se explican las enseñanzas características especiales de estas grandes unidades. Se trata de la eliminación de polvo y cuestiones de dosificación del combustible y del crudo, así como problemas del desgaste y de la conservación del precalentador de la chapa del horno y del enfriador. Se discute el consumo de calor teóricamente más económico y se muestran los problemas a resolver para ponerlo en práctica.

Esta disertación referente a los grandes hornos rotatorios muestra los caminos y los éxitos de la industria del cemento, en los últimos cuatro o cinco años, para poder fabricar económicamente sus productos, según los procesos modernos. La figura 1, relativa a la marcha de los precios de los materiales de construcción más importantes, en los últimos años, indica los esfuerzos que ha debido de realizar la industria del cemento para sostener, durante más de diez años, los mismos precios, a la vez que ha tenido que hacer frente a las cargas en el precio de coste, cuya magnitud puede deducirse de la consideración de la figura 2, que describe el desarrollo del valor del promedio de los componentes más importantes del coste y de la inversión requerida por trabajo humano. El salto en la producción de los hornos, de 600 a 1.000 y 1.200 t/día, unido a la racionalización de la economía térmica, impidieron la elevación de los costes. Por ello, han discurrido paralelamente estas dos preocupaciones: aumento en el tamaño de los equipos, orientado hacia la disminución del trabajo humano invertido, y esfuerzos para mejorar la economía térmica. El cuadro de valores 1 nos muestra, para la República Federal Alemana, la disminución en el número de los viejos hornos rotatorios por vía seca, con calcinadores o calderas para recuperación de calor perdido y el incremento en la aplicación de grandes hornos rotatorios por vía seca.

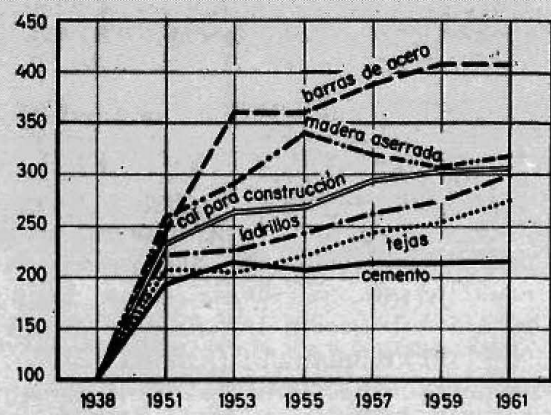

( ${ }^{\circ}$ Conferencia pronunciada en la Sesión de otońo de la Agrupación de Fabricantes de Cemento Alemanes.

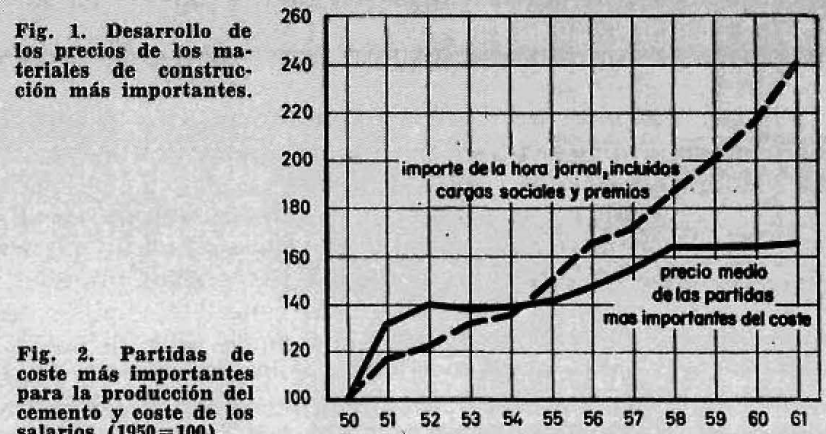

cemento y coste de los

$\begin{array}{llllllllllll}50 & 51 & 52 & 53 & 54 & 55 & 56 & 57 & 58 & 59 & 60 & 61\end{array}$ 
CUadro de valores 1. Desarrollo de los procesos modernos de calcinación.

$\begin{array}{lll} & 1954 & 1962 \\ \text { Hornos antiguos por vía seca .. } & 84 & 84 \\ \text { Hornos modernos por vía seca } & 34 & 79\end{array}$

La producción de los hornos presenta la tendencia creciente representada en la figura 3 , en tanto que el perfeccionamiento en la economía térmica hace reconocer los avances logrados por los proveedores de hornos.

La figura 4, que describe el desarrollo de la economía térmica de una de las fábricas de la República Federal Alemana desde el año 1950 , muestra lo que hay que conseguir, en casos particulares, con una racionalización consecuente y por el perfeccionamiento de la economía térmica. Con los precios actuales de los combustibles que, según el origen, se mueven alrededor de los $65 \mathrm{DM} / \mathrm{t}$ de carbón, el ahorro conseguido alcanza de 8 a $9 \mathrm{DM} / \mathrm{t}$ en el importe del coste técnico de producción $y$, sin esfuerzo alguno, se puede evaluar lo que significaría esta cifra si la industria del cemento y las firmas que fabrican hornos no hubieran avanzado por el camino que ha conducido a los grandes hornos rotatorios por vía seca, de explotación segura y de alto rendimiento térmico.
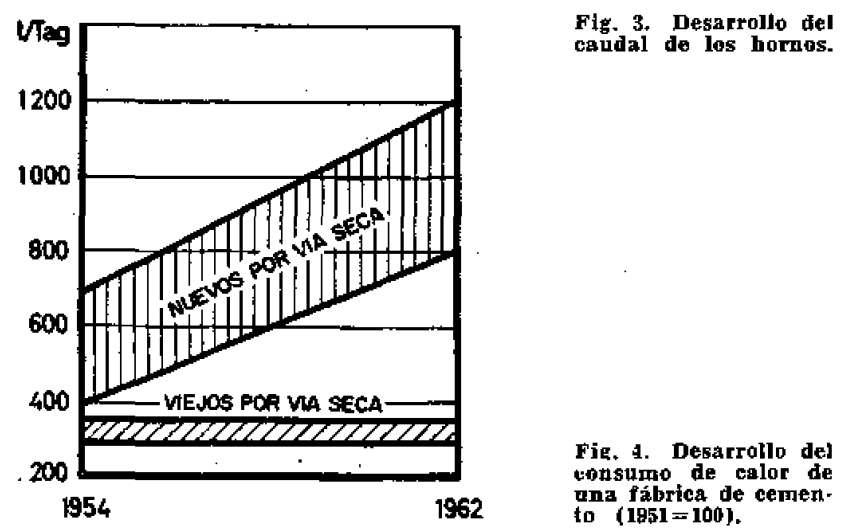
to $(1951=100)$

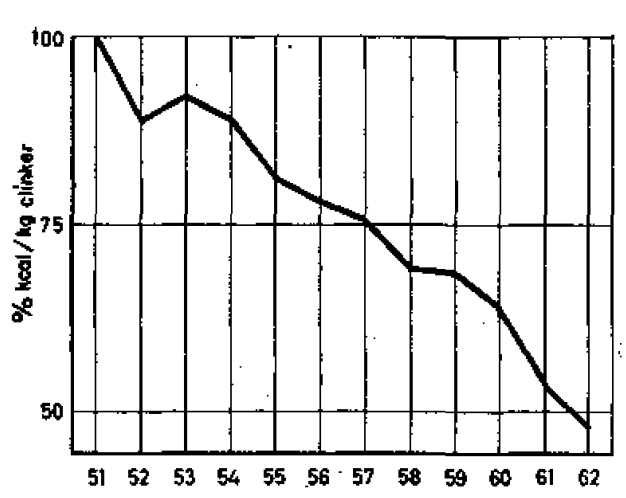

\section{Los hornos modernos por vía seca}

Los procesos que permiten la producción del clinker de cemento con un consumo bajo de calor pueden dividirse en dos grandes grupos: el procedimiento que aplica la parrilla, que también suele denominarse proceso semiseco, y el procedimiento por suspensión en gases, el cual es un proceso por via seca exclusivamente. La apiicación de la parrilla de calefacción previa, aparte de exigir la posibilidad de preparación de gránulos estables, sólo es aplicable, relativamente, a pocas circunstancias de materias primas-humedad, puesto que exige preparar primero la harina de crudo que ha de humedecerse de nuevo para preparar los gránulos. Esto no es necesario en el proceso por suspensión en gases, donde el crudo ya es introducido en el horno en forma de harina seca. Por esta razón se ha establecido la universalidad del procedimiento por suspensión en gases frente al de la aplicación de la parrilla. Todo lo que antecede se ha proyectado en el mercado, pues las ofertas para procesos por vía seca son, principalmente:

Procedimientos con parrilla: Polysius, que se ha introducido preponderantemente en la industria del cemento. Miag y Iurgi, para aplicaciones especiales.

Procedimientos por suspensión en gases: Smidth, Humboldt, Polysius, Krupp y Wedag, siguiendo la serie de su aparición, aunque el proceso Smidth no tenga ninguna unidad en explotación en la República Federal. La idea común a todos estos procesos consiste en el hecho de que su realización, que antes tenía lugar, de modo completo, dentro del horno mismo, se divide ahora de tal modo que los dos primeros estadios de la fabricación, a saber, la calefacción previa y la descarbonatación-por lo menos parcial-se efectúan en el sistema de precalentamiento (parrilla o ciclones). Los hornos mismos (parte rotatoria) pueden ser más cortos, aunque, sin embargo, en su aspecto constructivo permanecen como antes. Por tanto, para la descripción del proceso podemos limitarnos a los tipos de intercambiadores térmicos dispuestos delante del tubo rotatorio. 


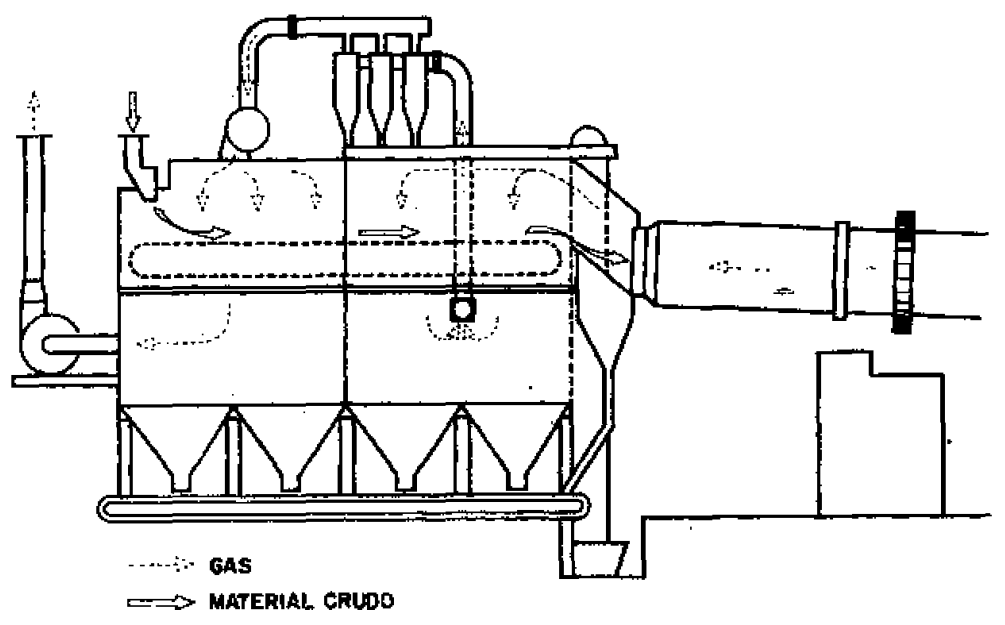

Fig. 5. Horno Lepol.

Fig. 6. Intercambjador Humboldt por suspensión en corriente gaseosa.

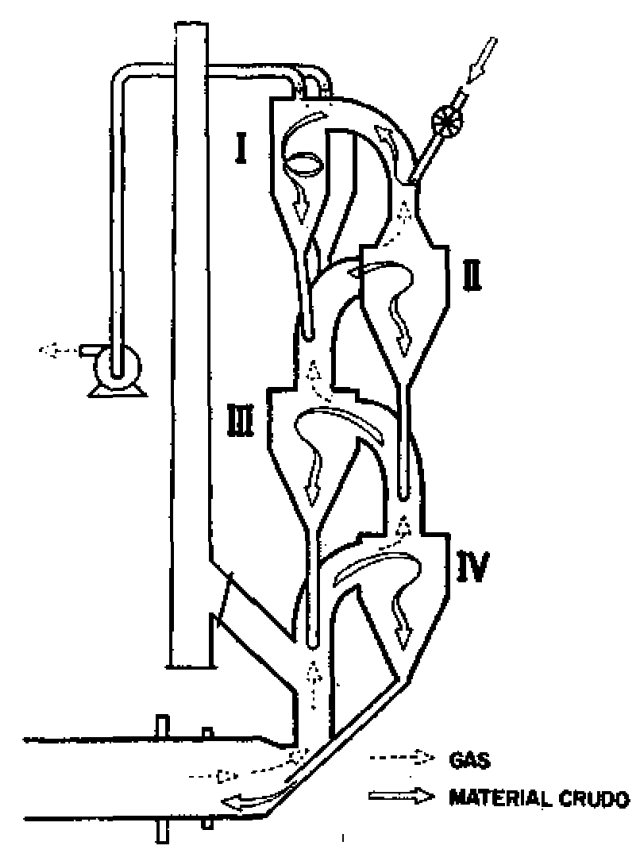

Puede darse por supuesto que es conocido el funcionamiento del proceso de parrilla representado por el horno Lepol, el más importante de aquéllos para la industria del cemento (fig. 5); además, ha poco, ha sido descrito exhaustivamente (1). Se dispone una parrilla móvil, de las del tipo corrientemente usado en hogares de calderas, que se divide en dos parte mediante una compuerta transversal, de modo que los gases del horno atraviesen, en serie, las dos cámaras de la parrilla mediante una llamada «doble circulación». Con ello los gránulos dispuestos sobre la parrilla son precalentados y descarbonatados parcialmente.

Las dificultades del proceso estriban, en realidad, en la preparación de los gránulos, pequeñas esferas de crudo que se disponen sobre la parrilla, en una capa de determinado espesor. En casi todos los casos se consigue la granulación del crudo con 10 y hasta 15 por 100 de agua. Sin embargo, no se tiene, ya con ello en la mano, el comportamiento de los gránulos sobre la parrilla. En él juegan un papel decisivo las propiedades de las materias primas y, además, la investigación acerea de la aptitud a la granulación de una harina de crudo es muy difícil. El principal fenómeno que dificulta la explotación de la parrilla Lepol es la explosión de los gránulos sobre aquélla, producida por la evaporación relativamente retardada del agua de granulación, euando la película exterior de los gránulos ya se ha endurecido, dando lugar a la destrucción de aquéllos; y le sigue en importancia el desgaste por rozamiento de los gránulos de superficie blanda. También puedert presentarse dificultades por la presencia simultánea de gránulos de tamaños desiguales.

Allí donde se posea la seguridad de contar con una buena preparación y conservación de los gránulos se obtendrá un clínker de características de excelente regularidad, por razón de la preparación previa de aquéllos. Las ventajas de este proceso son la alimentación regular de material al horno y ei avance, también regularizado, del material a lo largo de aquél.

Los procedimientos por suspensión del material en corriente gaseosa deben ser descritos con más detalle y a la vista de dibujos esquemáticos, puesto que el comportamiento del sistema gas-material es más complicado. Como en estos procedimientos tiene lugar un intercambio térmico entre el gas y el material suspendido en aquél, que es decisivo en todos ellos, se ha llegado a las formas de reálización más diversas como resultado de los esfuerzos efectuados para lograr la consecución de un rendimiento óptimo en el intercambio térmico.

El procedimiento Humboldt, de intercambio térmico por suspensión del material en los gases de combustión. ha encontrado la más amplia difusión (fig. 6). Humboldt dirige actualmente el montaje 


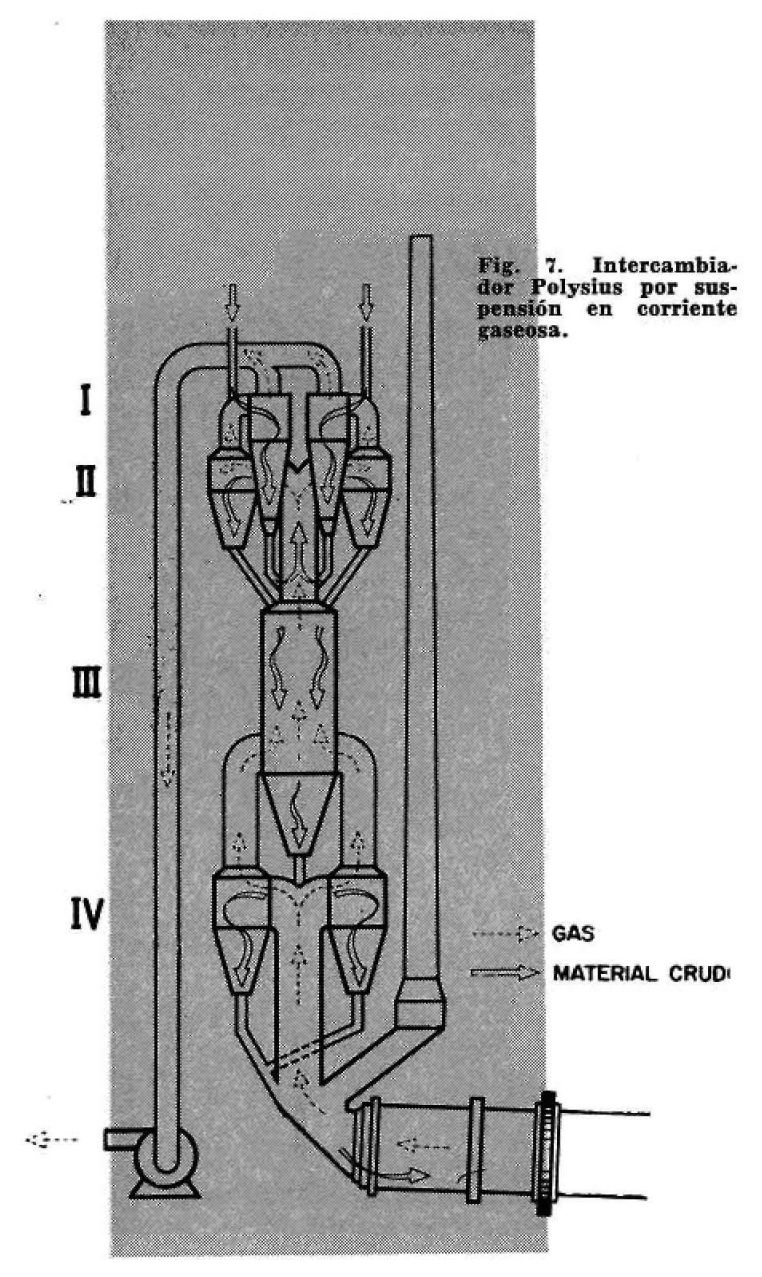

de la instalación núm. 100, con lo cual parece que se está bien documentado acerca de que sus instalaciones se han acreditado en gran escala. Son conocidas las torres características de este proceso, con los cuatro escalones de ciclones, de los cuales el más alto, es decir, el primer escalón considerado desde el punto de vista del flujo del material, está compuesto por dos ciclones, dispuestos en paralelo, con objeto de facilitar la operación de segregación del polvo contenido en la corriente gaseosa. Los gases de salida o residuales del horno circulan a lo largo de los ciclones de abajo arriba; el crudo frío es suministrado delante de los ciclones del primer escalón y arrastrado, a favor de la corriente, por la de los gases. El crudo, mediante la acción separadora de los ciclones, es segregado de la corriente gaseosa $y$, retrocediendo en su camino, vuelve a caer en la canalización de entrada de los gases del escalón anterior. Este juego se repite tres veces más y el material tiene, a la entrada del horno, una temperatura de alrededor de $50^{\circ} \mathrm{C}$ por encima de los 800 y está descarbonatado, aproximadamente en 1/3. La duración del recorrido del crudo a través de todo el intercambiador es de 20 a 30 segundos.

En este caso, los gases, frente al proceso de la parrilla, presentan a la salida del intercambiador, temperaturas de 330 a $360^{\circ} \mathrm{C}$, y quedan disponibles para ulterior utilización. En general, se calcula que con los gases residuales del horno Humboldt todavía pueden ser desecadas materias primas con un contenido de agua del 7 al 8 por 100. El proceso se hace particularmente económico, porque permite conseguir, empleando los gases residuales del enfriador, cuando se dan condiciones favorables, la desecación de materias primas con 10-12 por 100 de agua, sin aplicación de un hogar adicional. Como para el Lepol, las cifras de consumo de calor se mueven alrededor de las $800 \mathrm{kcal} / \mathrm{kg}$ de clínker, incluidas las cifras antes citadas para el secado. El clínker del horno Humboldt presenta un aspecto muy parecido al obtenido en hornos por vía húmeda con gradación de tamaños que alcanza hasta los 100 milímetros.

Polysius, en los últimos años, simultáneamente con su procedimiento de parrilla, ha desarrollado un intercambiador por suspensión en corriente gaseosa, al que ha llamado procedimiento «Dopol» (figura 7), porque la corriente gaseosa se subdivide en dos (doble corriente). Polysius, hasta ahora, ha construido cuatro instalaciones y ha recibido contratos para algunas más.

En este sistema llama la atención que, además del primer escalón, dispuesto como en el Humboldt, el segundo y cuarto también están constituidos por dos ciclones. Solamente el tercer escalón, llamado «recipiente turbillonario» (Wirbelschacht), está constituido por un solo elemento y tiene, sencillamente, el significado de un intercambiador de calor en contracorriente, en oposición a los escalones compuestos por ciclones. En esto radica la diferencia fundamental con el proceso Humboldt, y por esta causa quizás se consiga un intercambio térmico más activo. En un desarrollo ulterior, consecuente con esta idea, Polysius construye ya un sistema Dopol con dos escalones en contracorriente, con la intención de conseguir nuevas economías en el consumo de calor.

Es de señalar que la canalización de gases en el último escalón, el más caliente, es de sección elíptica. La razón para ello ha sido rebajar la formación de pegaduras, frente a la canalización de sección rectangular, así como hacer más fácil su limpieza. También resulta más favorable esta forma de sección, vista desde el ángulo de las condiciones técnicas para el movimiento de una corriente gaseosa. Para el vestido protector interior de la canalización se consigue también una estabilidad más elevada que en el caso de paredes planas. 
Se ha ideado un dispositivo para eliminar las pegaduras a la salida de los ciclones, que suelen presentarse muchas veces-lo que es bien sabido que ocurre en todos los procesos de este tipo-. Este dispositivo consiste en un sistema de toberas tangenciales servidas por aire comprimido y dispuestas, además, para funcionar automáticamente en cualquier momento.

La división de un sistema intercambiador de calor en dos equipos paralelos ofrece, en primer lugar, para la misma cantidad de gases, dimensiones más reducidas para los ciclones y mejor eficiencia en la segregación y, como consecuencia, caudales de circulación de polvo más reducidos. Además, se tiene la ventaja de que la obstrucción de una unidad del intercambiador es observable claramente por su diferente temperatura y permite resolver la avería a su debido tiempo. La marcha del crudo a lo largo del intercambiador, a excepción del escalón en contracorriente, es prácticamente la misma que en el Humboldt.

El prototipo de intercambiador térmico de tubos ascendentes Krupp ha sido instalado por la firma Dyckerhoff en la fábrica Mark I (figura 8). Una vez más el proceso consta de cuatro escalones; tres de ellos en forma de meandro (los números IV, III y II); el escalón tomado como número I consistía, inicialmente, en un multiciclón captador de polvo. En el curso del desarrollo de la instalación fue sustituido, siguiendo el ejemplo de los otros intercambiadores, por un escalón formado por un ciclón doble, ya que la carga de polvo de los gases era demasiado elevada.

En el interior de las canalizaciones del intercambiador «sinuoso» por las que ascienden los gases, van instalados dispositivos que dan origen a la deposición del polvo por acción centrífuga. Krupp pretende que, por la longitud relativamente grande de los tubos ascendentes, se alcance, prácticamente, la temperatura teórica de mezcla entre gases y material. Además, debe conseguirse una calcinación más avanzada por la prolongada permanencia del material en el aparato, ya que la calcinación no sólo es una cuestión de temperatura, sino también de tiempo. Además, se espera que debido a la verticalidad de los tubos se disminuya el peligro de las pegaduras, porque el material se fija, principalmente, en los tramos curvos. Las partes sobrecalentadas que poseen componentes con tendencia a producir pegaduras. en los tramos de canalización rectilínea, tienen ocasión de ser envueltas por capas de material menos fusible.

La recepción del material segregado se realiza, dentro de cada escalón, mediante un tubo que desemboca en la corriente gaseosa ascendente del escalón inmediato. De este modo se consigue una calefacción del crudo en el mismo orden de magnitud, aproximadamente, que para el sistema Humboldt o Polysius.

Mediante la canalización por tubos ascendentes y descendentes del intercambiador se consigue, como es bien comprensible, que la altura de la instalación sea menor que para las otras construcciones. Krupp quiere alcanzar con sus instalaciones de 780 a $800 \mathrm{kcal} / \mathrm{kg}$ de clínker.

Todavía no se dispone de valores obtenidos a través de una explotación de duración continuada; sin embargo, las mediciones realizadas en explotación durante varios días permiten vislumbrar que puede alcanzarse aquella meta. 


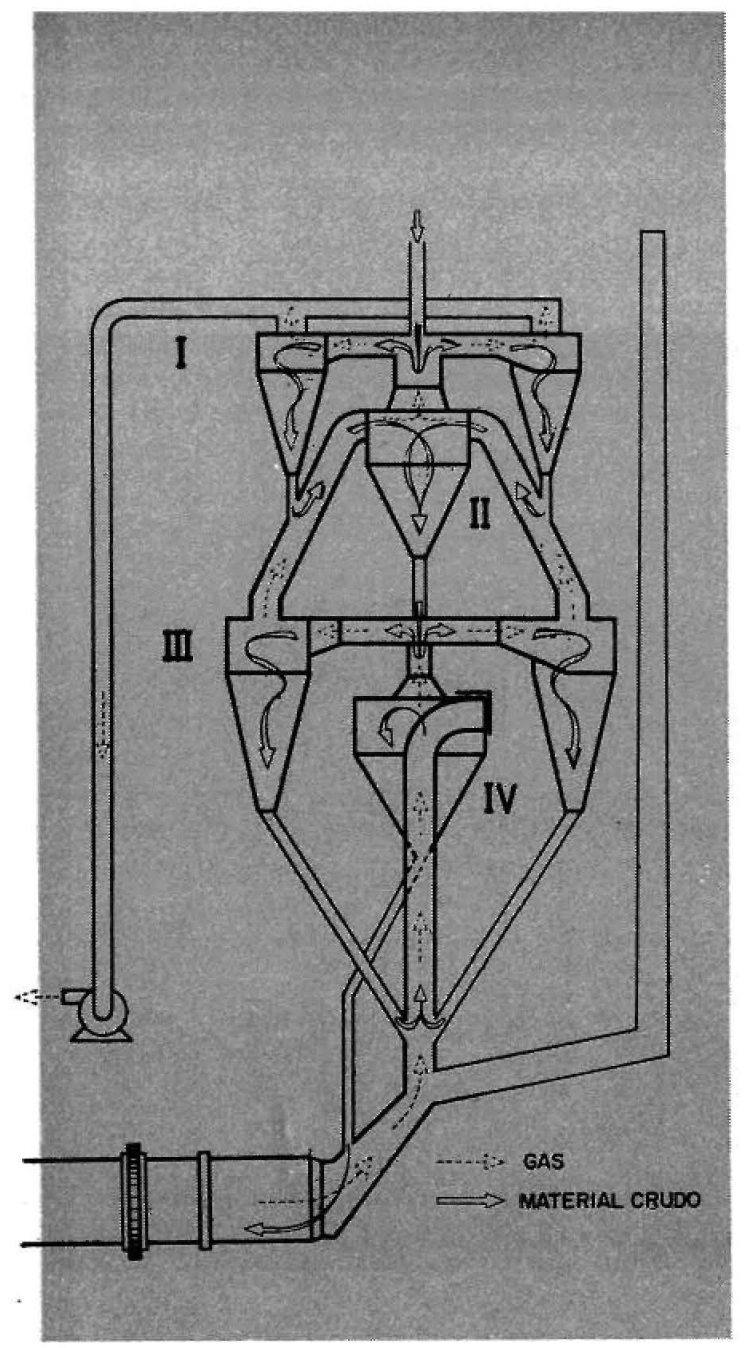

Fig. 9. Intercambiador Wedag por suspensión en corriente gaseosa.

El intercambiador térmico, por suspensión en corriente gaseosa, del tipo Wedag (fig. 9), trabaja, en todo caso, con cuatro escalones de ciclones, de los cuales el primero y el tercero están dispuestos como escalones dobles, mientras que los ciclones II y IV son llamados por Wedag ciclones principales. Entre los ciclones dobles han dispuesto los llamados «recipientes turbillonarios» (Wirbeltöpfe), en los que se pretende lograr un intercambio térmico en contracorriente, así como un movimiento turbillonario muy vivo e íntimo. También aquí se percibe la tendencia, por lo menos en algunos elementos, de trabajar en las condiciones más favorables, desde el punto de vista de la economía térmica, ofrecidas por el intercambio en contracorriente. Wedag escribe al explicar su instalación:

«Puesto que en el recipiente turbillonario o distribuidor se entretiene, de modo permanente, un movimiento de ese tipo, creado por el movimiento, en torbellinos, del gas dentro del ciclón, se eleva la permanencia del material en el seno de la corriente gaseosa $\mathrm{y}$, con ello, se consigue una transmisión de calor más favorable. Por razón de la salida tangencial de los recipientes turbillonarios, en los dos ciclones del escalón I se garantiza, a la par que la carga regular de los separadores, de acuerdo con la experiencia, una disminución de la pérdida de carga en el ciclón principal. El material depositado en los separadores del primer escalón, precalentado de 200 a $250^{\circ} \mathrm{C}$, es introducido en la canalización de gases que va del ciclón doble al escalón III, de tal modo, que es ofrecido el recorrido más largo posible hasta el ciclón II. A través de la larga permanencia así alcanzada, se compensa el intercambio térmico, menos eficaz, de la circulación en corriente paralela de material y gases, si se compara con el mecanismo de la transmisión en contracorriente. Desde el ciclón del escalón II, el material llega al recinto turbillonario II y es transportado con la corriente de gases correspondiente al escalón I a través del doble ciclón del piso III. Del ciclón principal IV, el crudo pasa al horno precalentado a unos $800^{\circ} \mathrm{C}$, y ya parcialmente descarbonatado.»

El consumo de calor está establecido en $800 \mathrm{kcal} / \mathrm{kg}$ de clínker. Wedag ofrece como ventajas de su procedimiento:

«Frente a los otros, esencialmente, menor resistencia de circulación de la corriente gaseosa, como consecuencia del tipo de montaje de los ciclones en paralelo y, con ello, menor inversión de energía en el ventilador. Disminución del peligro de pegaduras en los tubos ascendentes, motivada por la elevada velocidad y menor altura en la obra de construcción.»

La primera instalación entrará en servicio en los meses próximos en el Eifel; se trata, sin embargo, de una instalación pequeña de $400 \mathrm{t}$ /día.

\section{Resultados de explotación}

Después de la descripción de los tipos de intercambiador más importantes, quizás debemos hacer una consideración de los distintos balances térmicos (cuadro 2). Es necesario aclarar que en la evaluación de los balances térmicos se han tomado siempre en consideración dos cifras, que tienen su 
CUADRO DE VALORES 2 . Balances térmicos de grandes hornos rotatorios por vía seca.

Proceso con parrilla

Procesos con suspensión en corriente gaseosa

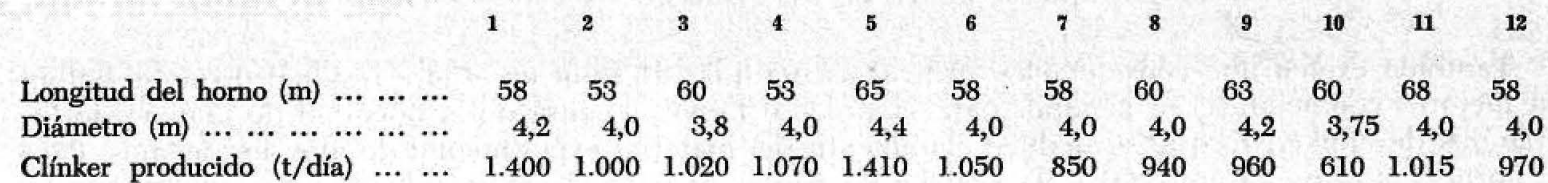

Balance térmico (kcal/kg clínker):

\begin{tabular}{|c|c|c|c|c|c|c|c|c|c|c|c|}
\hline Formación del clínker $\ldots . . . .$. & 400 & 400 & 400 & 420 & 410 & 390 & $-(\infty)$ & 400 & 410 & 415 & 415 \\
\hline Radiación y convección $\ldots \ldots \ldots$ & 70 & 50 & 70 & 60 & 40 & 65 & $-\left({ }^{\circ}\right)$ & 60 & 60 & 80 & 45 \\
\hline Pérdida en el clínker ... ... ....... & 15 & 25 & 15 & 15 & 25 & 20 & $-\left({ }^{\circ}\right)$ & 15 & 15 & 10 & 20 \\
\hline Polvo y resto $\ldots \ldots \ldots \ldots$ & 20 & 20 & 35 & 25 & 15 & 35 & $-\left({ }^{\circ}\right)$ & 35 & 5 & - & - \\
\hline
\end{tabular}

$\left({ }^{\circ}\right)$ Faltan detalles, valores de explotación.

origen en que al lado de la obtenida como resultante de una duración de explotación sin perturbaciones de 24-48 horas, ha habido siempre otra que corresponde a períodos de explotación más o menos perturbada. El valor que se refiere a este último caso es el más importante para la consideración de los costes y de los resultados de la explotación. Para estudios comparativos, sin embargo, para comenzar, debe contarse poco con ellas como punto de partida. La economía térmica puede estar influenciada, ciertamente, por pérdidas de rendimiento procedentes de la sección del clínker y por consumos más elevados de carbón por razón de los encendidos del horno; sin embargo, su importancia apenas puede tomarse en consideración como base para una comparación. Digamos todavía, que las cifras de consumo reducido pueden ser sobrepasadas en 30-50 unidades térmicas en una explotación corriente.

Es fácil ver que son distinguibles los balances, del procedimiento de la parrilla y el del intercambio por suspensión en corriente gaseosa, por las partidas de la evaporación de agua y de la pérdida en los gases residuales. Para las instalaciones Lepol los valores para evaporación de agua están comprendidos entre 115 y $145 \mathrm{kcal} / \mathrm{kg}$ de clínker, según la humedad de los gránulos; mientras que en el caso del intercambiador por suspensión en corriente gaseosa, esos valores son despreciables para nuestras consideraciones. En las pérdidas por gases residuales, para el intercambiador por suspensión, las cifras oscilan entre 130 y $175 \mathrm{kcal} / \mathrm{kg}$ de clínker, debido a su temperatura más alta frente a las del horno Lepol, que oscilan desde 40 a $65 \mathrm{kcal} / \mathrm{kg}$ de clínker.

Las pérdidas por radiación oscilan, según el estado del revestimiento refractario, aislado o no, de $40 \mathrm{kcal} / \mathrm{kg}$ de clínker, como valor mínimo, a $120 \mathrm{kcal} / \mathrm{kg}$ de clínker, como máximo. Cuando, a pesar de ese valor tan elevado, se consigue un consumo total de calor de $800 \mathrm{kcal} / \mathrm{kg}$ de clínker, se reconoce ya la posibilidad de una ulterior reducción de dicho consumo térmico. Los valores de las pérdidas por radiación presentan dispersiones que no dependen del tipo de proceso.

Debemos hacer ahora unas observaciones acerca del aislamiento de los hornos y el material aislante. Está ya firmemente establecido que los modernos hornos por vía seca deben estar aislados para mantener las pérdidas por radiación tan pequeñas como sea posible. Esto es válido, también, para los sistemas de precalentamiento, en lo que es posible, técnicamente, conseguir los valores mínimos. En consecuencia, hay unanimidad acerca del aislamiento de la zona de precalefacción y de la zona de calcinación. Se discute, tanto ahora como antes, el aislamiento de la zona de sinterización, la cual, según los resultados de una encuesta, debe probar a aislarse sólo cuando se presentan dificultades para la formación de costra en esta zona del horno. En la mayor parte de los casos existe una costra suficiente en la zona de sinterización y, entonces, el aislamiento de ésta no conduce a nada. Las experiencias con materiales de revestimientos aislantes de los distintos proveedores son muy dife- 
rentes. Las condiciones de explotación y las propiedades de las materias primas influyen mucho en 1 a estabilidad del revestimiento de ese tipo $y$, como es lógico, de modo más intenso que cuando se trata del revestimiento normal que se aplica en esas zonas.

También exigen una consideración crítica las pérdidas de calor en el aire residual, Estas se hallan en mejores condiciones, en general, para el proceso Lepol, a causa de la igualdad de tamaño de los gránulos de clínker, lo que se deduce claramente del material experimental de que disponemos. Para el horno Lepol oscila entre 60 y $95 \mathrm{kcal} / \mathrm{kg}$ de clínker y para el intercambiador por suspensión en corriente gaseosa entre 100 y $120 \mathrm{kcal} / \mathrm{kg}$ de clínker, si se prescinde de una excepción (la disposición «duotérmica» del enfriador).

Hay muchas influencias que frustran el efecto óptimo, a saber: eliminar las pérdidas por aire residual. Al lado de la desigualdad de tamaño de los gránulos de clínker, ya citada, juega un papel decisivo la distribución del clínker sobre la parrilla del enfriador, sobre la cual deben operar las cantidades de aire empleadas. La estabilidad de las placas es una magnitud más que da origen a perturbaciones $y$, así, la persona dedicada a la explotación se mueve entre distintas variables de influencia, $y$ tiene que ensayar para buscar las condiciones óptimas de trabajo.

El cuadro de valores 3 permite observar cómo varían las cantidades de aire empleadas para enfriar el clínker, desde $1,67 \mathrm{~m}^{3} \mathrm{~N} / \mathrm{kg}$ de clínker hasta $2,8 \mathrm{~m}^{3} \mathrm{~N} / \mathrm{kg}$ de clínker. Prescindiendo de la excepción antes citada, los valores más altos corresponden al intercambiador por suspensión en corriente gaseosa análogamente a lo dicho anteriormente.

CUADRO DE VALORES 3. Cantidades de aire para enfriamiento en los grandes hornos rotatorios por via seca.

\begin{tabular}{|c|c|c|c|c|c|c|c|c|}
\hline F A B R I C A & 3 & 4 & 5 & 6 & 8 & 10 & 11 & 12 \\
\hline 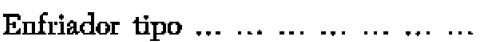 & 837 & 850 & 2.418 & 844 & 1.237 & 1.033 & 1.244 & 850 \\
\hline Distancia entre ejes $(m) \ldots \ldots \ldots$ & 11,40 & 15,30 & 18,60 & 13,35 & 11,40 & 10,10 & 13,35 & 15,30 \\
\hline 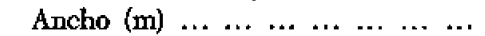 & 2,45 & 2,45 & 2,40 & 2,45 & 3,65 & 3,05 & 3,65 & 2,45 \\
\hline Cantidad de clínker $(t / d i a)$ :.. $\ldots \ldots$ & 1.020 & 1.070 & 1.410 & 1.050 & 940 & 610 & 1.015 & 970 \\
\hline Cantidad de aire $\left(\mathrm{m}^{3} \mathrm{~N} / \mathrm{kg}\right.$ de clinker) & 1,75 & 2,22 & 1,72 & 1,76 & 2,59 & 2,80 & 2,28 & 1,67 \\
\hline
\end{tabular}

Hace unos meses una fábrica suiza ha ensayado, con éxito, hacer marchar un enfriador conectado a una circulación de aire correspondiente solamente al aire secundario. Se hacía trabajar el enfriador con $1 \mathrm{~m}^{3} \mathrm{~N} / \mathrm{kg}$ de clínker, de aire para enfriamiento; la temperatura del clínker subía hasta $250-300^{\circ} \mathrm{C}$ y las pérdidas de calor por el aire residual quedaban en $50 \mathrm{kcal} / \mathrm{kg}$ de clínker. Pero como se conseguía trabajar sin pérdida alguna de calor a causa del aire residual del enfriador, se llegaría a un consumo total de calor de $700 \mathrm{kcal} / \mathrm{kg}$ de clínker. No se dispone todavía de datos numéricos exactos por la corta duración de la explotación. Es cierto que tales temperaturas en el clínker supone instalar detrás del enfriador transportadores de acero; sin embargo, este modo de trabajar sería más económico en todo caso, puesto que así no habría đe hacerse ninguna inversión para la utilización del aire residual.

Las otras partidas del balance térmico se mueven entre valores habituales: los calores de formación del clínker, entre 390 a $420 \mathrm{kcal} / \mathrm{kg}$ de clínker; el calor perdido en el clínker, de 10 a $25 \mathrm{kcal} / \mathrm{kg}$ de clínker; y la partida por pérdidas en el polvo y resto, entre 5 y $35 \mathrm{kcal} / \mathrm{kg}$ de clínker.

Establecer ia utilización de los calores residuales tropieza, parcialmente, con dificultades importantes. En este punto, es sabido, que el «abono en cuenta» no ha sido usado para los hornos, ya que verdaderamente es un vicio rebajar «ópticamente», mediante manipulaciones de cálculo, el consumo bruto de calor aplicado. A continuación surge el estudio del problema de si se tienen que hacer inversiones más cuantiosas para aprovechar el calor residual de baja calidad, suplementado con calor más noble, para el secado de las materias primas, o si se ha de contar con resultados más favorables aplicando una calefacción aparte, que ofrezca consumos específicos de calor más bajos para la evaporación del agua. Además, únicamente en casos muy raros hay concordancia entre la utilización del calor residual y el caudal de producción del homo; es decir, en general, se secan, a veces, menores, 
y con más frecuencia, mayores cantidades de materias primas que las exigidas por la marcha del horno en un instante dado. $Y$, por añadidura, los gases disponibles exhiben temperaturas bajas que requieren su elevación mediante una calefacción adicional, lo que hace más complicada todavía la circunstancia.

La dificultad del problema estriba en las bases defectuosas de su planteamiento. Por consiguiente, en el cuadro 4 se ha procedido de tal modo que en la primera línea se ha colocado la cantidad de calor necesaria para la evaporación del agua contenida en las materias primas, calculada a partir de su humedad solamente; en la segunda se ha inserito el valor del calor residual utilizado partiendo de

CUADRo DE VALORES 4. Utilización del calor re sidual de Ios grandes hornos por via seca.

\begin{tabular}{|c|c|c|c|c|c|}
\hline & & & Intercamb & por su & \\
\hline $\begin{array}{lllllll}\mathbf{F} & \mathbf{A} & \mathbf{B} & \mathbf{F} & \mathbf{I} & \mathbf{C} & \mathbf{A}\end{array}$ & 3 & 4 & 7 & 11 & 12 \\
\hline Longitud del horno $(m) \ldots \ldots \ldots \ldots \ldots \ldots \ldots \ldots$ & 60,0 & 53,0 & 58,0 & 68,0 & 58,0 \\
\hline 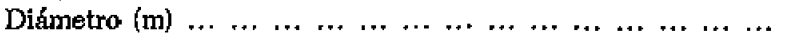 & 3,8 & 4,0 & 4,0 & 4,0 & 4,0 \\
\hline Cantidad de clinker $(t /$ día $) \ldots \ldots \ldots \ldots \ldots \ldots$ & 1.020 & 1.070 & 850 & 1.015 & 970 \\
\hline Cantidad de gases $\left(\mathbf{m}^{3} \mathrm{~N} / \mathrm{kg}\right.$ de clinker $) \ldots \ldots \ldots \ldots \ldots$ & 2,08 & 1,82 & - & 1,58 & 1,57 \\
\hline 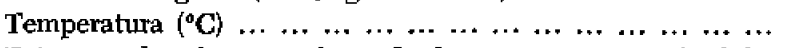 & 89 & 125 & 340 & 322 & 325 \\
\hline $\begin{array}{r}\text { Exigencia de calor para el secado de materias primas }(\mathbf{k c a l} / \mathrm{kg} \\
\text { de clínker) } \ldots \ldots \ldots \ldots \ldots \ldots \ldots \ldots \ldots \\
\end{array}$ & 82 & 56 & 120 & 140 & 185 \\
\hline Calor residual utilizado del horno, etc. (kcal $/ \mathrm{kg}$ de clínker). & 95 & 80 & 170 & 170 & 245 \\
\hline 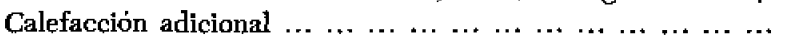 & sí & sí & no & no & no \\
\hline $\begin{array}{llllllllllllll}\text { Caudal de secado } & \ldots & \ldots & \ldots & \ldots & \ldots & \ldots & \ldots & \ldots & \ldots & \ldots & \ldots & \ldots & \ldots\end{array}$ & 1,5 & 2,6 & $\begin{array}{l}1,0 \\
\text { más carbón }\end{array}$ & 1,2 & 1,2 \\
\hline Humedad de las materias primas $(\%) \ldots \ldots$ & 3,5 & 2,8 & $4-6$ & 7,5 & 9,7 \\
\hline
\end{tabular}

lo ofrecido por los hornos, o sea, el contenido por el aire residual del enfriador, por el intercambiador, o por la suma de ambos; en la línea siguiente se ha fijado si hay o no calefacción adicional de los gases; y el caudal del secadero o del molino-secadero, expresado como múltiplo de las necesidades de secado exigidas por el caudal de producción del horno, en la penúltima línea. Con esto se han sorteado los cálculos y aclaraciones circunstanciales que, en este resumen, son poco interesantes, aunque lo sean mucho para cada instalación en particular.

Considerando el material numérico anterior se llega a expresar con claridad la delimitación de la zona de aplicación, citada anteriormente, para la parrilla o el intercambiador por suspensión en corriente gaseosa, si se añade en el cuadro 4 la última línea en la que se indican las humedades de las materias primas; hasta el valor máximo de 3,5 por 100 de humedad es aplicable el proceso de parrilla para las instalaciones consideradas, y por encima de ese valor hay que aplicar una calefacción adicional; mientras que las materias primas fuertemente humedecidas son el campo de aplicación típico para el caso con intercambiador en suspensión gaseosa, pues pueden trabajar sin calefacción adicional utilizando el calor residual de los gases del horno y del aire residual del enfriador, aun con un caudal de secado superior al requerido por el de producción del horno.

Los datos contenidos en el cuadro 5 , relativos a los consumos específicos de energía eléctrica en las instalaciones investigadas, provocarán la protesta de varias firmas constructoras de hornos.

CUADRo DE VALORES 5. Consumo especifico de energía de los grandes hornos rotatorios por vía seca.

\begin{tabular}{|c|c|c|c|c|c|c|c|c|}
\hline \multirow[b]{2}{*}{ F A B B I C A } & \multicolumn{3}{|c|}{ Parrilla } & \multicolumn{5}{|c|}{ Intereambiador por suspensión } \\
\hline & 2 & $\mathbf{s}$ & 4 & 7 & 8 & 18 & 11 & 12 \\
\hline Longitud del homo $(m) \ldots \ldots \ldots \ldots$ & 53,0 & 60,0 & 58,0 & 58,0 & 60,0 & 60,0 & 68,0 & 58,0 \\
\hline 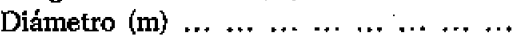 & 4,0 & 3,8 & 4,0 & 4,0 & 4,0 & 3,75 & 4,0 & 4,0 \\
\hline $\begin{array}{l}\text { Cantidad de clínket } \ldots \ldots \ldots \ldots \ldots \ldots \ldots \\
\left.\text { Consumo específico de energía }{ }^{(}\right)\end{array}$ & 1.000 & 1.020 & 1.070 & 850 & 940 & 610 & 1.015 & 970 \\
\hline$(\mathrm{kWh} / \mathrm{t}) \ldots \ldots \ldots \ldots \ldots \ldots$ & 12,5 & 12,2 & 12,6 & 22,0 & 13,2 & 21,9 & 21,5 & 19,7 \\
\hline
\end{tabular}

(*) Incluido desde la alimentación de crudo hasta la cadena de arrastre del enfriador con la totalidad de las instalaciones de depuración de gases. 
Las cifras son valores de explotación y contienen todo lo que hoy forma parte de uu horno, a partir de la aitmentación de material, desde el silo de crudo, hasta la cadena de arrastre de debajo del enfriador, con todas las instalaciones de deposición de polvo, tanto por parte de la sección del horno como del enfriador. No tiene sentido dar cifras comparativas, sin citar la eliminación de polvo, puesto que sin estas instalaciones, tomada en cuenta la parte correspondiente al enfriador, no puede marchar ningún horno. Aparte de esto, está claro que las inversiones para las instalaciones de deposición de polvo se expresan en energía, de acuerdo con el grado de depuración deseado; además, no se han tenido en cuenta en los consumos específicos las generosidades en el dimensionado de los motores y las reservas establecidas que, aunque no demasiado, elevan algo aquellos valores.

Si consideramos los números del cuadro en particular, tenemos también caracterizados los dos grandes grupos de procesos; así, el intercambiador por suspensión en la corriente gaseosa se caracteriza por el gran consumo del exhaustor colocado detrás del intercambiador y el elevado consumo requerido por la eliminación de polvo. Por eso, los homos Lepol muestran consumos entre 12,2 y 12,6 $\mathrm{kWh} / \mathrm{t}$ de clínker, y entre 19,7 y $22 \mathrm{kWh} / \mathrm{t}$ de clínker los hornos con intercambiador por suspensión en corriente gaseosa, con lo cual sólo queda sin aclaración el valor del horno número 8.

\section{Comportamlento en explotación e informaciones particulares}

Por el comentario de los balances térmicos resalta ya de qué modo tan decisivo pueden actuar las perturbaciones en la explotación sobre la economía. Por tanto, deben estar esquematizadas las circunstancias características de la explotación y también ciertas informaciones especiales. El origen de las perturbaciones puede referirse, en ambos procedimientos, a las combinaciones cloruradas de los álcalis, las cuales, en el proceso de la parrilla o del intercambiador por suspensión en corriente gaseosa, pueden conducir a dificultades.

En el proceso de parrilla son conocidas las pegaduras en la zona de transición de horno a parrilla, cuando no se retiran del circuito el polvo de los eiclones o los polvos cargados de álcalis de los filtros electrostáticos. En el intervalo de 1 a 4 semanas las pegaduras han de ser destruidas y, por tanto, la instalación ha de permanecer parada durante unas horas. Esporádicamente aparecen algunas pegaduras sobre las placas de la parrilla, cuyo origen no se conoce todavía.

En el intercambiador se debe contar con el depósito, cuando se sobrepasa el límite del 3 por 100 , de cloruros en la parte inferior del último ciclón. Es fácilmente demostrable el enriquecimiento en cloro y en álcalis, en el crudo de alimentación, antes de llegar a la entrada del horno. Sin embargo, se presentan intervalos en los cuales, con porcentajes esencialmente elevados de cloruros, las instalaciones siguen marchando sin ninguna perturbación. La eliminación de las pegaduras y la posibilidad de ser evitadas puede conseguirse por interrupción del circuito, con objeto de eliminar los componentes clorurados alcalinos como ha descrito Mussgnug (2). En todo caso, la solución representa un empeoramiento de la economía térmica y únicamente en casos excepcionales debe tenerse en cuenta. Los intercambiadores exigen un control sistemático y una vigilancia en cada escalón para no dejar aparecer, de ninguna manera, pegaduras en las zonas peligrosas. Estas costras son el origen de las obstrucciones en las canalizaciones del crudo, pues se resuelven en tortas que al desprenderse caen en aquéllas. El descubrir demasiado tarde una obstrucción representa una grave dificultad con todas sus consecuencias desagradables $y$, quizás por ello, tienen ciertas ventajas los sistemas que trabajan con dos ciclones en paralelo, porque se producen distribuciones asimétricas de temperatura sobre las cuales se debe estar atento. Es preciso tener en cuenta que en el caso de perturbaciones por obstrucción se debe suspender tajantemente el trabajo, ya que por sobrecalentamientos del crudo se puede dar lugar a que se produzcan pegaduras de mayor espesor todavía.

Por esta misma razón se debe marchar con una buena dosificación en la alimentación del crudo, puesto que, al tener lugar una carga variable en la corriente gaseosa, pueden producirse sobrecalentamientos locales que activan la formación de pegaduras. Debiera ser perfectamente comprensible que la constancia en las condiciones de los gases de salida del horno, así como la regularidad de marcha del mismo, exigen una dosificación del combustible. También es válido, que en el Lepol sea deseable la instalación de básculas dosificadoras; sin embargo, no son necesarias de modo obligatorio, pero su ausencia exige una elevada atención por parte del encargado del granulador. 
La dificultad más grave para los enfriadores de parrilla es la eliminación de las «setas», estalagmitas que se producen sobre el altar del enfriador originando un trabajo pesado para el personal de servicio, con la perturbación simultánea para la economía térmica, porque hacen descender la temperatura del aire secundario cuando las «setas» han de ser destruidas a mano. Las máquinas para producir golpes para destruirlas no conducen, a veces, a los resultados deseados, en particular cuando ya se ha formado una «seta». Son más efectivos los resbaladeros enfriados por agua circulante, que son rentables por la elevación de caudal que por ellos se consigue por explotación ininterrumpida. Cuestan de 10 a $.15 \mathrm{kcal} / \mathrm{kg}$ de clínker.

Algo debiera decirse también acerca de los problemas de deposición de polvo. Es sabido que las circunstancias para la eliminación del polvo son favorables al proceso con parrilla, a causa de la pequeña carga de polvo en los gases y a la reducida cantidad de éstos. Lo demuestran los valores de orientación de los costes de instalación de los filtros electrostáticos; los órdenes de magnitud son:

Para procesos por vía húmeda, aproximadamente: $355 \mathrm{DM} / \mathrm{m}^{3}$ de gas a depurar, que corresponde a $2,00-3,50 \mathrm{DM} / \mathrm{t}$ año.

Para los procesus con parrilla, aproximadamente: $5-8 \mathrm{DM} / \mathrm{m}^{3}$ de gas a depurar, que corresponde a $1,60-2,50 \mathrm{DM} / \mathrm{t}$ año.

Para los intercambiadores por suspensión en corriente gaseosa, aproximadamente: $8-10 \mathrm{DM} / \mathrm{m}^{3} \mathrm{de}$ gas a depurar, que corresponde a $2,50-3,00 \mathrm{DM} / \mathrm{t}$ año.

La situación de explotación «directa» en el proceso con intercambiador por suspensión en corriente gaseosa, es decir, sin utilización de los gases residuales en el secadero o en una instalación de molienda y secado simultáneo, presenta siempre problemas, puesto que supone canalizaciones de desviación estancas; de lo contrario, se llega a fuertes entradas de aire falso, en intervalos de temperatura que hacen difícil o impiden en absoluto el efecto de eliminación de polvo. Ciertamente, que siempre se está preocupado, en el sentido de conseguir una economía térmica racional, por hacer que estos intervalos de trabajo sin secadero sean cortos, pero en explotación es inevitable que se produzcan y exigen la introducción de variantes en forma de inyección de agua mediante toberas, o en resolver constructivamente mejor los órganos de cierre de las canalizaciones.

Del entretenimiento de estos equipos debemos decir que presentan un desgaste normal. Los hornos de hoy son mucho más estables y los cojinetes de apoyo se realizan de manera que, normalmente, no presentan ninguna molestia. El desgaste de los segmentos de la boquilla de salida es distinto según la posición đel dardo de la llama. El desgaste del revestimiento, en casos desfavorables, es del orden de $0,2 \mathrm{~kg} / \mathrm{t}$ para la zona de sinterización, y alrededor de $0,7 \mathrm{~kg} / \mathrm{t}$ para el resto de la obra de ladrillo; por tanto, su importe es de 0,50 DM/t de clísker.

El desgaste en la parrilla del enfriador presenta esencial importancia en las placas de aquélla; desgaste que es más alto para el intercambiador por suspensión en corriente gaseosa que para el proceso de parrilla, por las razones ya conocidas. Conocemos enfriadores que dan lugar a la sustitución de 1 a 2 placas al mes como promedio, y también casos límites en que el promedio es la sustitución de 1 placa diaria, lo que significa un coste de $0,25-0,20 \mathrm{DM} / \mathrm{t}$ de clínker. Otro punto débil para el enfriador es la cubierta colgada, cuyo enlace con la caja del enfriador puede dar lugar a molestias si, por Ilegar a ella polvo, se producen compresiones en los hombros.

La parrilla Lepol tiene, en general, muy poco desgaste. Se calcula en 0,02 hasta $0,08 \mathrm{DM} / \mathrm{t}$ de clínker para placas de la parrilla, tepuestos del ventilador y cosas parecidas. En el intercambiador no hay, prácticamente, ningún gasto de conservación si se ha realizado bien la disposición del refractario. El desgaste de los ventiladores y de los otros dispositivos mecánicos es de un orden de magnitud favorable, como en el caso de la parrilla; en todo caso, hasta 0,08 DM/tonelada.

La inversión de personal debe ser considerada de igual cuantía para la parrilla y el intercambiador, $y$ no debe ser inferior a tres hombres para un equipo de $1.000 \mathrm{t}$, en consideración del valor de la instalación; a saber: un vigilante para el intercambiador o para el granulador, un hornero y un vigilante para el enfriador. Este servicio existe en todas las instalaciones en alguna for- 
ma, sólo que muchas veces se les denomina de otro modo. Los puestos de mando centralizados no producen grandes variaciones en el resultado final; y si se miden las perturbaciones que son posibles por falta de vigilancia sobre el valor de las instalaciones representado por millones, resultan sostenibles los $0,30 \mathrm{DM} / \mathrm{t}$ de clínker por inversión de personal.

Se comprende que es imprescindible tener al alcance de la mano todos los medios auxiliares modernos de regulación y vigilancia de estas grandes instalaciones. Los cuadros para vigilancia, realizados en forma bien madurada, con multiplicidad de indicaciones de temperatura, presión y de caudales, con o sin contactos para valores límite, con inscrippción continua para los datos más importantes. pertenecen al utillaje necesario. La velocidad angular del ventilador principal, así como la del horno. han de ser regulables en intervalos muy amplios. En los ventiladores se aplica cada vez más el motor de corriente trifásica con rotor en cortocircuito con un reductor hidráulico; para el accionamiento del horno se aplican, recientemente, al lado de máquinas asíncronas con regulación por resistencias y las máquinas de conmutación, motores de corriente continua con rectificadores de silicio que se comportan bien. Los procesos con parrilla se equipan con distintas regulaciones, con las cuales se mantienen constante las cantidades de aire residual, la presión en el cabezal de horno y la diferencia de presiones en las cámaras de la parrilla. En los intercambiadores suelen ser suficientes, en general, los dos primeros de los ciclos de regulación. Sobre esto se debe ser explícito en el sentido de que, con la exactitud de la dosificación y la homogeneidad del crudo y del combustible, las regulaciones llegan a ser menos necesarias. Para circunstancias dificiles de las materias primas, sin embargo, deben existir los distintos ciclos de regulación por representar la regularidad del producto a obtener.

Para la elección del tipo de horno correcto no hay ninguna receta general, puesto que las distintas influencias juegan su papel en cada caso parícular. Pueden darse sólo algunos puntos de vista válidos en sentido general. Así, se dará preferencia al proceso con parrilla cuando la humedad de los materiales llegue hasta quizá el 5 por 100 como máximo, si al mismo tiempo se está seguro de que por las condiciones de las materias primas permiten preparar unos gránulos estables, con objeto de conseguir con ello las ventajas de un clínker de Lepol muy regular, que crean la posibilidad de condiciones muy favorables de trabajo en el enfriador.

El intercambiador por suspensión en corriente gaseosa debe ser introducido siempre cuando la humedad en las materias primas se encuentra por encima del 5 por 100 , para poder utilizar en el secado de aquéllas las 140 hasta $170 \mathrm{kcal} / \mathrm{kg}$ de clínker de que se dispone detrás del intercambiador. Además, debe ser propuesto el intercambiador en todos los casos en que se presenten condiciones deficientes en los gránulos, traducidos en forma de explosiones, destrucción por rozamiento, etc. Pero en este caso se ha de contar con clínker irregular, que se parecerá más o menos al clínker de los hornos por vía húmeda.

En casos extremadamente raros se pueden predecir qué dificultades se presentarán, según el proceso aplicado. Desde ese punto de vista no se está seguro ni con el proceso con parrilla ni con el de intercambiador, tanto en sentido positivo como en negativo. En las particularidades, ya hemos planteado antes estas cuestiones de modo muy somero.

\section{El consumo de calor teóricamente más favorable}

En los años próximos pasados se han propuesto, de modo permanente, perfeccionamientos en la economía térmica. Sería interesante fijạr adónde se puede llegar como resultado final. Por consiguiente, debe ser planteada la cuestión de dónde debe ser situado el límite, teóricamente alcanzable, para el consumo de calor. De modo inverso, por razón de las exigencias de los procesos de alta temperatura fue supuesto que se establecía un límite inferior de consumo de calor, el cual estaba por encima, de modo importante, de la cantidad de calor exigida por la teoría; por ejemplo, 650 kcal. Para los sistemas de enfriador instantáneo debía ocurrir lo mismo; por tanto, los perfeccionamientos ulteriores dependerán de la exigencia de crear otro enfriador, por ejemplo el enfriador vertical (Schachtkühler). Un camino en esa dirección es también la introducción intensiva de los intercambiadores en contracorriente.

El consumo teórico de calor para la cocción del clínker, así como las partidas que aparecen como calores cedidos en nuestros balances térmicos, es sabido que se definen como diferencias de entalpía 
a $20^{\circ} \mathrm{C}$, entre el crudo, por un lado, y el clínker, $\mathrm{CO}_{3}$ y vapor de agua (de la arcilla), por el otro. Tal consumo depende de la composición del crudo; pero esencialmente del contenido en CaO y está comprendido entre los límites de 380 a $450 \mathrm{kcal} / \mathrm{kg}$ de clínker. Algunos colegas son de la opinión de que se puede llegar a valores muy próximos a este límite teórico en el proceso de la vía seca pura. Naturalmente, que los dispositivos de calcinación deberían de ser considerados según circunstancias distintas de las de ahora. Con estas constataciones se quiere mantener vivo el pensamiento de los constructores de hornos, a partir de los puntos de vista de la teoría; la tendencia al consumo de calor más favorable no debe tener un límite en las 700 a 650 kcal. Ahora bien, lo bonito para los teóricos es que ellos se lo imaginan todo y que no necesitan preocuparse de la realización de los problemas que ellos proponen, y así, en los últimos tiempos, han aparecido repetidamente trabajos que anuncian, pasito a pasito, un posible descenso ulterior del consumo del calor. Con el balance térmico, que damos a continuación, que concluye con un consumo de 550 kcal (cuadro 6), se presenta un balance que no está en contraposición con la termodinámica, y que representa, ciertamente, para los constructores prácticos de hornos y con los puntos de vista actuales, un tema muy difícil.

Se ha introducido un consumo teórico relativamente alto de $430 \mathrm{kcal} / \mathrm{kg}$ de clínker. La pérdida por las paredes del sistema en conjunto se ha fijado en $60 \mathrm{kcal} / \mathrm{kg}$ de clínker, valor alcanzado ya prácticamente. La pérdida de calor en el clínker cón $20 \mathrm{kcal} / \mathrm{kg}$ de clínker, y $40 \mathrm{kcal} / \mathrm{kg}$ de clínker como pérdida en los gases residuales, son también valores que están establecidos en los balances térmicos.

\section{CUADRo DE VALORES 6. Límite teórico del consumo de calor téoricamente alcanzable.}

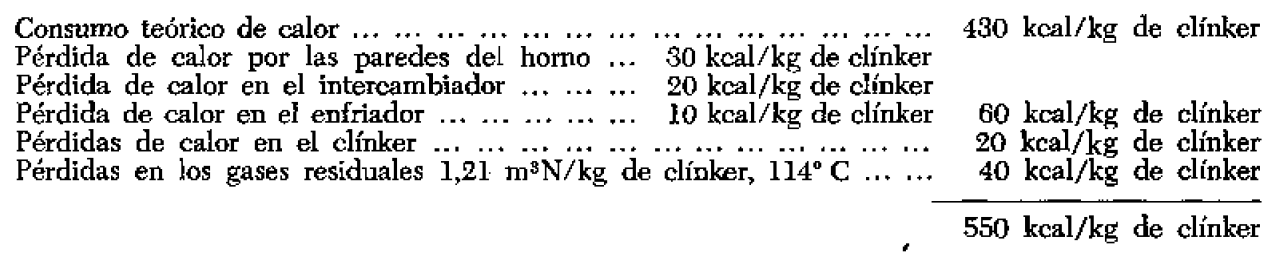

Aire de combustión, $0,892 \mathrm{~m}^{*} \mathrm{~N} / \mathrm{kg}$ de clínker $(n=1,5)$.

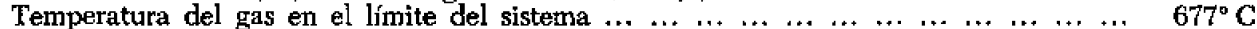

Temperatura del material en el límite del sistema $\ldots \ldots \ldots \ldots \ldots \ldots \ldots \ldots \ldots \ldots, 550^{\circ} \mathrm{C}$

$127^{\mathrm{n}} \mathrm{C}$

Para poder recuperar el calor del clínker después de la zona de sinterización debe ser aceptada una dilución para el aire de $n=1,5$, lo que representa una cuantía para el aire de combustión de alrededor de $0,9 \mathrm{~m} \cdot \mathrm{N} / \mathrm{kg}$ de clínker. La diferencia de temperatura entre gases y material en el límite del sistema se ha calculado en $127^{\circ} \mathrm{C}$. Las pérdidas por gases residuales resultan de una cantidad de gases de $1,21 \mathrm{~m}^{2} \mathrm{~N} / \mathrm{kg}$ de clínker a 114 grados centígrados.

Sí estos deseos y esperanzas se convierten algún día en hechos, dependerá, de un modo muy amplio, de los avances en la técnica de construcción de máquinas.

En el presente trabajo tomaron parte activa señores de la Agrupación Alemana de Cemento, las fábricas que han participado dando a conocer datos y discutiendo su material de investigación y su experiencia, y mis colegas Dr. Hummel y Dr. zur Strassen, por la discusión de los fundamentos téóricos. A todos ellos se lo agradezco desde aquí muy cordiaimente.

\section{Bibliografía}

(1) Weislehner, G.: Der Lepolofen in der Zementindustrie VDI--Nachrichten, 1922, núm. 22.

(2) Mussgnug, G.: Beitrag zur Alkalifrage in Sclwebegas-wirmetauscheröfen, 7ement-Kalk-Gips, 15 (1962), $197-204$. 